\title{
Melorheostosis: A Case Report in Pediatric Age Group
}

\author{
Rakesh Mehra $^{1}$, Richa Bhartiya ${ }^{2 *}$ and Pallavi Agrawal ${ }^{2}$ \\ 'Department of Radiology, Patna Medical College \& Hospital (PMCH), Ashok Rajpath, Patna, Bihar, India \\ ${ }^{2}$ Department of Pathology, Patna Medical College Hospital (PMCH), Ashok Rajpath, Patna, Bihar, India
}

\begin{abstract}
Melorheostosis is a rare, benign, and disabling condition. It is a rare mesenchymal bone disease classified as a sclerotic bone dysplasia. We present this case because of its rarity and highlight the importance of Fine needle aspiration cytology which is a minimally invasive technique in ruling out the possibilities of osteomyelitis and malignancies. Fine Needle Aspiration Cytology (FNAC) negates the use of unwarranted biopsies. The disease is a benign entity with a generally chronic course and periods of exacerbation and remission. Therapy is symptomatic and is aimed at controlling pain.
\end{abstract}

Keywords: Sclerotic Bone Dysplasia, Cytology, Biopsy, FNAC

\section{Introduction}

Melorheostosis is a rare disorder characterized by mesodermal dysplasia of bone first described by Leri and Joanny in 1922. ${ }^{[1]}$ It occurs sporadically with an incidence of 0.9 in 1 million with equal sex distribution. It usually occurs in the limbs and frequently crosses synovial joints. The etiology is unknown. ${ }^{[2]}$ We present the case of a 14-yearold female diagnosed with localized melorheostosis in the middle third of tibia. This case is presented to emphasize upon the awareness of the lesion to avoid unnecessary biopsy done to diagnose this case. It can be simply diagnosed on the basis of clinical and radiological features with a correlation with various laboratory investigations. Fine needle aspiration cytology proves to be an effective tool in ruling out the various infective and malignant differential diagnosis.

\section{Case Report}

A 14-year-old female presented with continuous pain in the middle third of tibia. No skin atrophy or erythma of the affected limb was detected. However, there was a diffuse bony prominence below the tibeal tuberosity. There was no restriction of movement of the limb or any soft tissue contracture. There was no other bony involvement. She was advised a simple X-ray, Fine needle aspiration cytology (FNAC) of the lesion and various blood tests. Ultrasound guided FNAC was performed which yielded bloody aspirate. Smears examined showed blood and blood elements. No malignant cell was identified. There was no evidence of inflammation in the form of sheets of polymorphs or lymphocytes or necrosis. No fungus or any other organism was identified. The cytological aspirate was also sent for microbiological culture which was negative for aerobes, anaerobes, typical and atypical mycobacteria and fungi. Based on the above findings possibility of osteogenic sarcoma, osteomyelitis and any fungal or bacterial infection was ruled out. X-Ray of the left leg presented with hyperostosis and thickening of the cortex of the middle third of tibia extending along the bone and showing a dripping candlewax image (Fig 1). Various laboratory investigations included complete blood count, calcium, phosphate, bony alkaline phosphatase, thyroid hormone profile and proteins. All these levels were within normal limits. She had normocytic normochromic anemia with a moderately elevated C-reactive protein of $60 \mathrm{mg} / \mathrm{L}$ (normal $<10 \mathrm{mg} / \mathrm{L}$ ). Based on the radiological and laboratory investigations she was diagnosed as a case of melorheostosis. She was started on treatment with analgesics and NSAIDS to which she responded very well.

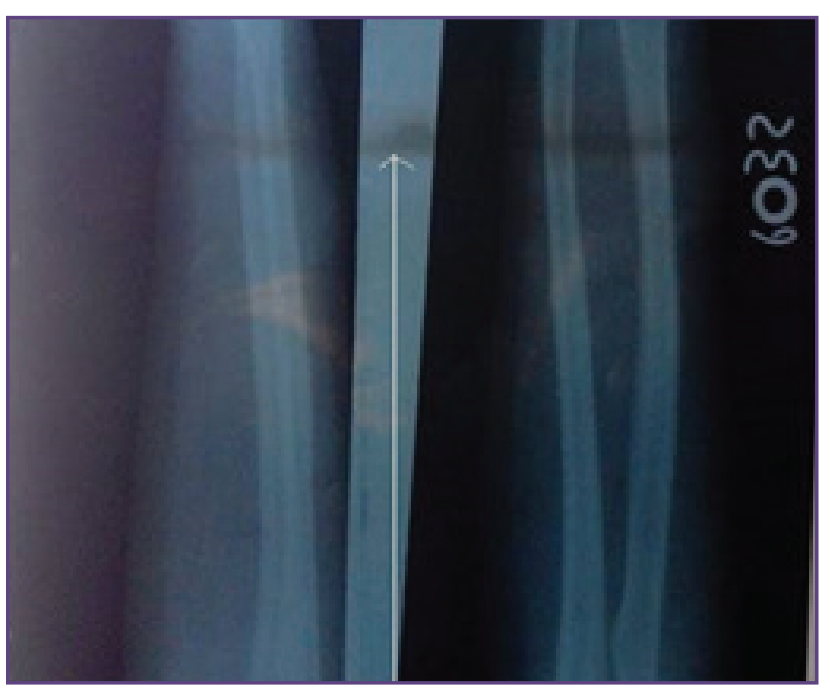

Fig. 1: Radiograph showing the tibia of 14 year old girl who had melorheostosis 


\section{Discussion}

Melorheostosis is derived from the Greek melos, meaning limb and rheos means flow. It is mixed sclerosing dysplasia with disturbance of both enchondral and intramembranous ossification, in which disordered intramembranous ossification dominates. ${ }^{[3]}$ There is wide range of age distribution from 2-64 years with equal sex predilection. The patients can present with various symptoms including pain, limb swelling, restricted range of motion of extremity due to soft tissue contracture. Other symptoms include stiffness, swelling, numbness, tingling and carpal tunnel syndrome. Lower extremities are more frequently involved. Other sites involved are skull, facial bones, ribs, scapula, pelvic bone and spine. Involvement can be monostotic, polyostotic or monomelic. Other changes include pigmentation, erythma, odema, periarticular fibrosis, weakness and atrophy of muscles, perivascular fibrosis with obliteration of blood vessels. Vascular lesions associated with melorheostosis include hemangiomas, vascular nevi, varices, glomus tumors, arteriovenous malformations and aneurysms. It has also been associated with tumors, including osteogenic sarcoma, malignant fibrous histiocytoma and dermoid tumors. Other associations are neurofibromatosis, tuberous sclerosis, scleroderma, rheumatoid arthritis and hypophosphatemic rickets. ${ }^{[4]}$

The etiology of this disorder is unknown. It is hypothesized that it may be due to vascular insufficiency or failure in intra-membranous or enchondral ossification. Freyshimidt $^{[5]}$ suggested that it is a form of mosaicism rather than early embryonic infection of the sensory nerve, in which the disorder could be due to the action of a lethal gene that survives only in mosaic state. Other theories propose a vascular disorder, inflammation, a degenerative lesion of the connective tissue, and embryonic damage as etiopathogenic factors. Recent studies have reported loss of function mutations in the LEMD3 gene, encoding an inner nuclear membrane protein that influences Smadsignalling, as a cause of osteopoikilosis, Buschke-Ollendroff syndrome and melorrheostosis. ${ }^{[6]}$

The classic radiographic features of melorheostosis are regular and wavy hyperostosis referred to as flowing candle wax appearance. Radiologically, five patterns have been described- classical, osteoma-like, myositis ossificans-like, osteopathiastriata-like and mixed type. There is usually a distinct demarcation between the affected bone and normal bone. Most typically the outer bony cortex is affected, but extension into the cancellous bone is also seen. In children, the hyperostosis is endosteal, marked by streakiness of the long bones and spotting of the small bones, whereas in adults it is in an extracortical, subperiosteal location. Four types have been described depending on the clinical situation: monostotic, polyostotic, monomelic and with generalized skeletal involvement. Diaphysis of long bones is more commonly involved. Other sites include the pelvis and bones of hands and feet. The ribs and the craniofacial complex are affected least often. CT and MRI are more useful for assessment of lesions involving the axial skeleton. ${ }^{[5,7]}$

Laboratory abnormalities reported in association with bone and soft tissue lesions of melorheostosis affect osteoblastic specific factor-2 (osf-2), osteonectin, fibronectin, transforming growth factor- $\beta$, and fibroblastic growth factor-23 (FGF-23). Serum calcium, phosphorus, and alkaline phosphatase levels have been reported to be within normal limits in melorheostosis. ${ }^{[8]}$

Histologic findings include variable degree of cortical thickening consisting of chondroid islands surrounded by woven or non-lamellar dense bone depending on stage of maturation with thickened, sclerotic, and irregular lamellae. An adjacent zone of fibrocartilage may show irregular surface fibrillation. A large quantity of osteoid without mineralization suggests over-production of bone matrix in affected bone. Increased osteoclasts reflect increased bone resorption. This indicates that increased bone formation and bone resorption are combined processes in melorheostosis. Rarely, presence of a cartilage cap over a portion of the sclerotic bone in intra-articular melorheostosis, could lead to misinterpretation of osteochondroma. Cells immunopositive for TGF $\beta$ and bFGF are densely present in melorheostotic bone. Both of these cytokines are osteogenic and angiogenic, probably these cytokines play a role in increased bone formation and increased angiogenesis. Increased expression of procollagen alpha1 mRNA expression and alpha1 (1), alpha2 (1), and alpha (111) collagen secretion has been observed in dermal fibroblasts obtained from a skin biopsy overlying the involved bone. Overall histology is nonspecific, but is used to exclude malignancy. Moreover FNAC can also serve this purpose so minimally invasive techniques should be applied to rule out other causes. ${ }^{[9]}$

Various differential diagnosis include myositis ossificans, osteopetrosis, osteopoikilosis, chronic osteomyelitis, parosteal and periosteal osteosarcoma, calcium pyrophosphate dihydrate (CPPD), deposition disease, osteoma, and Caffey's disease. Rare differentials include calcified synovial sarcoma, extraskeletal osteosarcoma, or tumoralcalcinosis. ${ }^{[3,4,7]}$

The treatment is limited and includes surgical interventions in the form of tendon lengthening, excision of soft tissue masses, release of joint contractures and occasionally amputation. Primary aim of treatment is pain relief and 
restoration of full range of motion. Conservative measures include analgesia, manipulation, braces, sympathectomy, physiotherapy, nerve blocks and serial casting. In some cases, good results are obtained with infusions of zoledronic acid. ${ }^{[10]}$ The prognosis is variable and depends on the anatomical location, extension into the soft tissues, and soft tissue changes. Melorheostosis does not shorten life span, however morbidity may be considerable. The disease exhibits a slow, chronic course with periods of exacerbation and arrest. Recurrence usually is expected after operative excision. ${ }^{[4]}$

\section{Conclusion}

To conclude melorheostosis is benign in nature with chronic pain in which deformity can be debilitating. FNAC is an effective measure to rule out various differential diagnoses of malignant and infective lesions. Biopsy should be avoided. Findings should be correlated with characteristic radiological features. Surgical intervention is advocated in chronic debilitating symptoms. Early diagnosis and effective treatment can translate these lesions into near complete resolution of the symptoms.

\section{Reference}

1. Leri A, Joanny J. Une affection non decrite des os: hyperostose "en coulee" surtoute la longueur d'un membreou "melorheostose". Bull MemSoc Med Hosp Paris. 1922; 46:1141-1145

2. Resnick D. Diagnosis of bone and joint disorders. $3 \mathrm{rd}$ ed, Vol. 6. Philadelphia, PA: WB Saunders; 1995;4410-3.

3. Greenspan A. Sclerosing bone dysplasias-a target-site approach. Skeletal Radiol. 1991; 20(8):561-583

4. Jain VK, Arya RK, Bharadwaj M, Kumar S Melorheostosis: Clinicopathological Features, Diagnosis, and Management. Orthopedics 2009;32(7)

5. Freyschmidt J. Melorheostosis: a review of 23 cases EurRadiol, 2001;11:474-479

6. McDermott M, Branstetter BF, Seethala RR. Craniofacial melorheostosis J Comput Assist Tomogr, 2008;32:825-827

7. Monte TC, Sala DR, Rubió JB, Bittermann V, Abelló JC. Melorheostosis, a Case Report

8. Reumatol Clin.2011;7:346-8

9. Sureka B, Mittal MK, Udhaya KK, Sinha M, Mittal A, Thukral BB. Melorheostosis: Two atypical cases. Musculoskeletal Radiology 2014;24(2):192-195

10. Hoshi K, Amizuka N, Kurokawa T, Nakamura K, Shiro R, Ozawa H. Histopathological characterization of melorheostosis. Orthopedics. 2001; 24(3):273-277.

11. Hollick RJ, Black A, Reid D. Melorheostosis and its treatment with intravenous zoledronic acid. BMJ Case Rep. 2010 doi: 10.1136/bcr.04.2009.1757

*Corresponding author:

Dr. Richa Bhartiya, \% -Shri Vinay Kumar Shrivastava, Dy. CSTE, Bungalow No. 882, Railway Officers' Colony, Danapur

(KHAGAUL) - 801105, District - Patna, Bihar, India

Email: richabhartiya1972@gmail.com

Date of Submission : 14.12.2016

Date of Acceptance : 02.06.2017

Financial or other Competing Interests: None.

Date of Publication : 11.10.2017 